\title{
miR-92a promotes tumor growth of osteosarcoma by targeting PTEN/AKT signaling pathway
}

\author{
JIE XIAO ${ }^{1}$, WEIFENG YU ${ }^{1}$, KONGZU HU ${ }^{2}$, MAOQIANG LI ${ }^{3}$, JIANWEI CHEN ${ }^{4}$ and ZHANCHUN $\mathrm{LI}^{4}$ \\ ${ }^{1}$ Department of Anesthesiology, Renji Hospital, School of Medicine, Shanghai Jiaotong University, Shanghai 200127; \\ ${ }^{2}$ Department of Orthopaedic Surgery, The First Affiliated Hospital of Anhui Medical University, Hefei, \\ Anhui 230022; ${ }^{3}$ Department of Orthopaedic Surgery, Hangzhou First People's Hospital, Hangzhou, \\ Zhejiang 310006; ${ }^{4}$ Department of Orthopaedic Surgery, Renji Hospital, School of Medicine, \\ Shanghai Jiaotong University, Shanghai 200127, P.R. China
}

Received September 28, 2016; Accepted February 20, 2017

DOI: $10.3892 /$ or.2017.5484

\begin{abstract}
MicroRNAs (miRNAs) play critical roles in human cancers including osteosarcoma (OS). miR-92a has been found to be a cancer-related miRNA in many cancer types and it is upregulated in OS cell lines. However, the expression and biological function of miR-92a in OS have not been investigated. In this study, we showed that miR-92a expression was increased in OS tissues, and its high expression was correlated with clinical stage, $\mathrm{T}$ classification and histological differentiation. Furthermore, patients with high expression of miR-92a had a significantly poorer survival rate. Functionally, miR-92a overexpression promoted the proliferation and cell cycle progression, and inhibited apoptosis in MG-63 cells. While inhibition of miR-92a showed contrary effects with reduced proliferation, cell cycle arrest at G1 phase and increased apoptosis in U2OS cells. Moreover, we confirmed that miR-92a inhibition reversed the tumor growth of OS cells in nude mice. Phosphatase and tensin homolog (PTEN), a well-known tumor suppressor, was confirmed to be the direct downstream target of miR-92a in OS. Notably, miR-92a consequently regulated the expression of the downstream targets of PTEN/AKT signaling pathway including p-Akt(Ser473), mTOR, p-p27(Thr157) and p-MDM2(Ser166). Furthermore, PTEN knockdown abrogated the functional effects of miR-92a silencing on the proliferation, apoptosis and cell cycle progression in OS cells. Thus, miR-92a that exerts an oncogenic role by targeting PTEN/AKT pathway in OS potentially acts as a biomarker and drug-target.
\end{abstract}

Correspondence to: Dr Zhanchun Li, Department of Orthopaedic Surgery, Renji Hospital, School of Medicine, Shanghai Jiaotong University, 1630 Dongfang Rd., Shanghai 200127, P.R. China E-mail: lzctgzyyx@163.com

Key words: miR-92a, osteosarcoma, cell proliferation, cell cycle, apoptosis, PTEN/AKT pathway

\section{Introduction}

Osteosarcoma (OS) is a very common malignant bone tumor especially in children and young adults (1), accounting for approximately $19 \%$ of the malignant cancer in the bone. Although some advances have been achieved in the treatment of OS including surgery, chemotherapy and radiotherapy, the long-term prognosis of OS patients remains poor $(2,3)$. It is urgent to identify novel biomarkers and therapeutic targets for OS which will greatly improve the prognosis of OS patients. Therefore, it is of extreme importance to clarify the molecular mechanisms underlying the pathogenesis of OS.

MicroRNAs (miRNAs), a group of non-coding, short (14-22 nucleotides in length) RNAs (4), play fundamental roles in human diseases by interacting with 3'-UTR of targeted mRNA and inhibiting the translation of targeted genes (5). They are involved in various biological processes (6) including cell differentiation, proliferation, apoptosis and movement. Moreover, increasing evidence showed that by acting as either oncogenes or tumor suppressors, deregulated expression or function of miRNAs is a critical reason for the development and progression of a number of human cancers $(5,7-9)$ including OS (10). Elucidating the expression and function of specific miRNA in OS will potentially lead to the identification of novel biomarkers and therapeutic targets in OS. miR-92a that belongs to miR-17-92 cluster is identified as a biomarker for pancreatic cancer, gastric cancer, prostate cancer, colorectal cancer and hepatocellular carcinoma (11-14). miR-92a facilitates the tumor growth of human hepatocellular carcinoma by targeting F-box and WD repeat domain-containing 7 (FBXW7) (14). Otherwise, miR-92a promotes the metastasis of nasopharyngeal carcinoma by targeting the phosphatase and tensin homolog (PTEN)/AKT pathway (15). In OS, Gougelet et al identified miR-92a as predictive tool, which was overexpressed in good responders to ifosfamide (16). Upregulated miR-92a was observed in OS cell line versus bone (17). However, the expression of miR-92a and its function in OS remain poorly disclosed.

In this study, we found that miR-92a expression was significantly increased in OS tissues. Increased expression level of miR-92a was correlated with malignant clinical features and 
reduced survival rate. Functionally, miR-92a could inhibit the tumor growth of OS by regulating cell proliferation, apoptosis and cell cycle progression. In addition, PTEN was found to be the direct downstream target of miR-92a. Furthermore, we confirmed that miR-92a exerted its biological functions by modulating PTEN/AKT signaling pathway in OS cells.

\section{Materials and methods}

Clinical tissues from OS patients. A total of 68 OS and 20 normal bone tissue samples were obtained from Renji Hospital, School of Medicine, Shanghai Jiaotong University during the period from January 2006 to December 2008. The demographic features and clinicopathologic data are shown in Table I. All patients signed the informed consents to participate in this study. The protocols and consents were approved by the Ethics Committee of Shanghai Jiaotong University and complied with the Declaration of Helsinki.

Cell lines and cell culture. The human OS cell lines used in this study were MG-63 and U2OS. These OS cell lines were obtained from the American Type Culture Collection (ATCC, Manassas, VA, USA). Cells were cultured in DMEM (Gibco, Grand Island, NY, USA) supplemented with 10\% fetal bovine serum (FBS, Gibco), streptomycin (100 $\mu \mathrm{g} / \mathrm{ml}$; Sigma, St. Louis, MO, USA), and penicillin (100 U/ml, Sigma).

Cell transfection. The miR-92a mimics and scrambled vector, miR-92a inhibitor and negative control inhibitor were obtained from GenePharma (Shanghai, China). PTEN siRNA and scramble siRNA were from Geneopenia (Guangzhou, China). Cell transfections in OS cells were carried out with Lipofectamine 2000 (Invitrogen, Carlsbad, CA, USA) according to the manufacturer's instructions.

RNA extraction and $q R T-P C R$. RNA from tissue samples and cell lines were extracted using TRIzol (Ambion, Austin, TX, USA). Real-time PCR for miR-92a was performed using TaqMan microRNA assays with specific primers for miR-92a. The PCR amplification and the quantification of the PTEN and GAPDH were performed with the ABI PRISM 7300 Sequence Detection System (Applied Biosystems, Foster City, CA, USA) and the SYBR ${ }^{\circledR}$ Premix Ex Taq ${ }^{\mathrm{TM}}$ II kit (Takara Bio, Shiga, Japan). The primers for PTEN, miR-92a, U6, GAPDH were from GenePharma. U6 was used as internal control of miR-92a while GAPDH was used as internal control of PTEN.

Cell proliferation assay. OS cells transfected with miR-92a mimic or inhibitor were seeded in 96-well plates and were incubated with CCK-8 (10\% diluted in normal culture medium, Dojindo; Kumamoto, Japan) at $37^{\circ} \mathrm{C}$ for $2 \mathrm{~h}$. Absorbance was measured at $0,24,48,72$ and $96 \mathrm{~h}$ after transfection at the wavelength of $490 \mathrm{~nm}$.

Cell cycle assays. At $48 \mathrm{~h}$ after the transfection, OS cells were collected and fixed overnight at $4{ }^{\circ} \mathrm{C}$ with $80 \%$ ethanol. Then, cells were incubated with propidium iodide (PI, Sigma) for $20 \mathrm{~min}$ at room temperature. Then, flow cytometry assays for OS cell cycle were carried out with a FACS Calibur (BD Biosciences, Bedford, MA, USA).
Table I. Correlation between the clinicopathological characteristics and miR-92a expression in osteosarcoma.

\begin{tabular}{|c|c|c|c|c|}
\hline \multirow[b]{2}{*}{ Clinicopathological features } & \multirow[b]{2}{*}{$\mathrm{n}$} & \multicolumn{2}{|c|}{$\begin{array}{l}\text { miR-92a } \\
\text { expression }\end{array}$} & \multirow[b]{2}{*}{ P-value } \\
\hline & & High & Low & \\
\hline Gender & & & & 0.324 \\
\hline Male & 40 & 18 & 22 & \\
\hline Female & 28 & 16 & 12 & \\
\hline Age (years) & & & & 0.177 \\
\hline$<24$ & 49 & 22 & 27 & \\
\hline$\geq 25$ & 19 & 12 & 7 & \\
\hline Clinical stage & & & & $<0.001^{\mathrm{a}}$ \\
\hline I & 27 & 8 & 19 & \\
\hline II & 34 & 20 & 14 & \\
\hline III & 7 & 6 & 1 & \\
\hline $\mathrm{T}$ classification & & & & $0.003^{\mathrm{a}}$ \\
\hline $\mathrm{T} 1$ & 26 & 7 & 19 & \\
\hline $\mathrm{T} 2$ & 42 & 27 & 15 & \\
\hline M classification & & & & 0.690 \\
\hline M0 & 61 & 30 & 31 & \\
\hline M1 & 7 & 4 & 3 & \\
\hline Histology & & & & 0.171 \\
\hline Conventional osteosarcoma & 58 & 31 & 27 & \\
\hline Others & 10 & 3 & 7 & \\
\hline Histological differentiation & & & & $0.026^{\mathrm{a}}$ \\
\hline G1 & 27 & 9 & 18 & \\
\hline $\mathrm{G} 2$ & 41 & 25 & 16 & \\
\hline
\end{tabular}

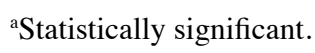

Cell apoptosis assay. The apoptosis of OS cells after transfection were examined with Annexin V/PI kit (BD Pharmingen, San Diego, CA, USA). OS cells were incubated with Annexin V/PI at $37{ }^{\circ} \mathrm{C}$ for 30 min and were subjected to a FACS Calibur (BD Biosciences) to evaluate the apoptotic rate.

In vivo experiments. $\mathrm{BALB} / \mathrm{c}$ nude mice were employed to perform nude mouse xenograft model. MG-63 cells transfected with miR-92a inhibitors or control cells were injected subcutaneously into the flank of the nude mouse. Tumor volume was determined every 3 days by measuring the length and width of the tumors and was calculated as tumor volume $=$ length $\mathrm{x}$ width $\mathrm{x}$ width $/ 2$. The animal protocols were approved by the Institutional Animal Care and Use Committee of Shanghai Jiaotong University.

Western blot analysis. Cellular proteins extracted from OS cells with RIPA buffer were separated by SDS-PAGE (4-20\%) and were then transferred to PVDF membranes (Amersham, Buckinghamshire, UK). Membranes were blocked with 5\% 
A

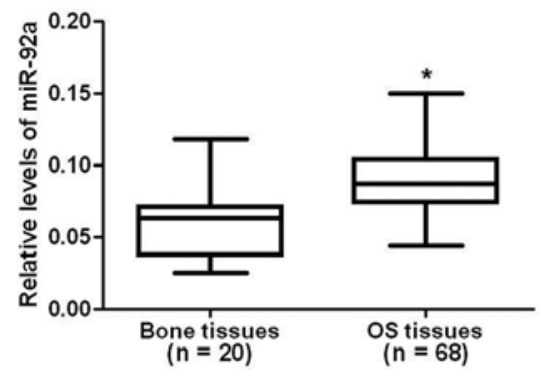

B

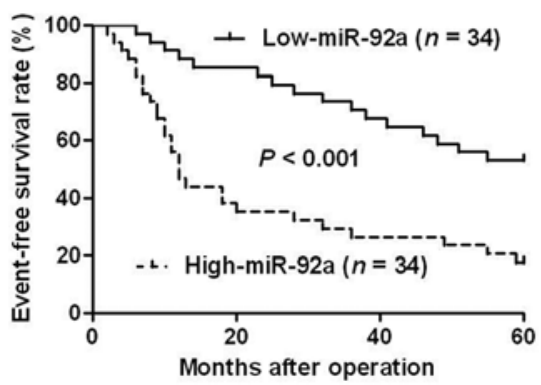

C

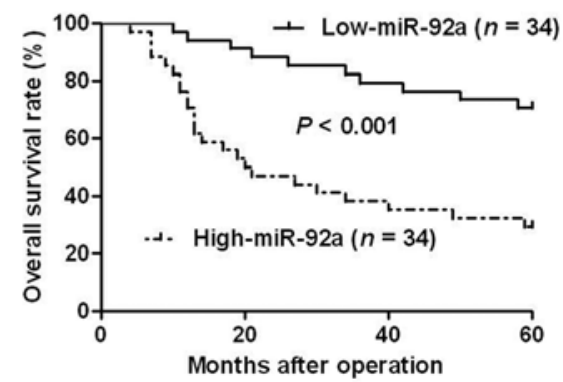

Figure 1. The expression level and prognostic value of miR-92a in OS. (A) miR-92a expression was increased in OS tissues as compared with normal bone tissues. ${ }^{*} \mathrm{P}<0.05$ by t-test. (B) Patients with high miR-92a level had significantly reduced event-free survival. $\mathrm{P}<0.05$ by Log-rank test. (C) Patients with high miR-92a level had significant poor overall survival. $\mathrm{P}<0.05$ by Log-rank test.

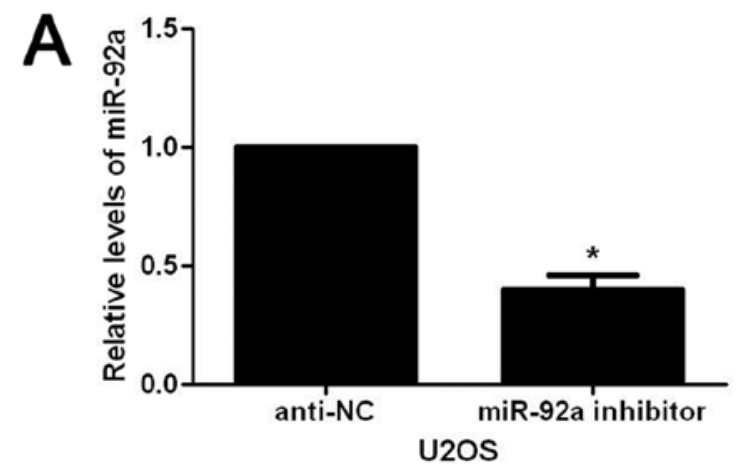

C

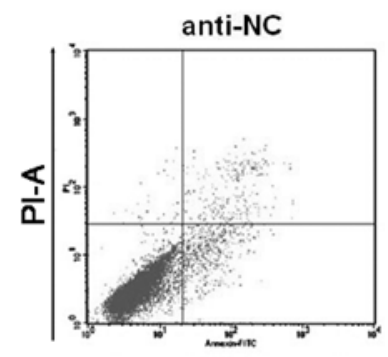

FITC-A
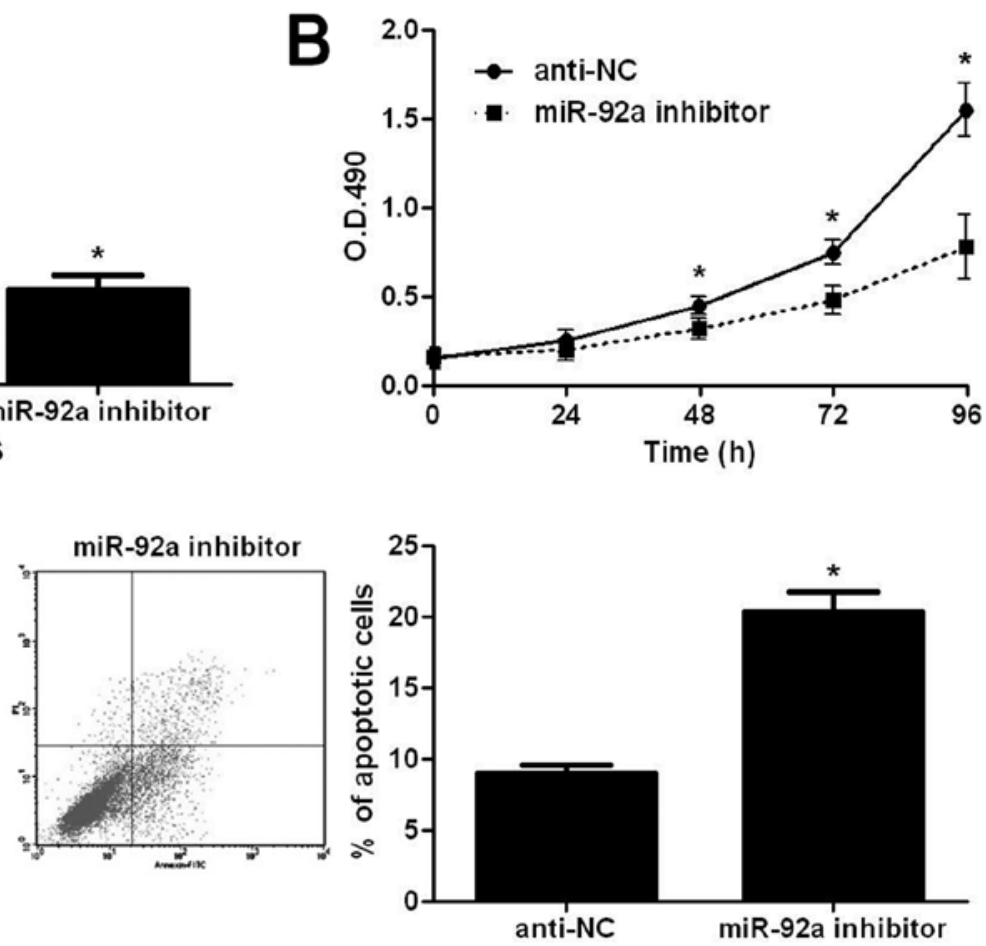

D
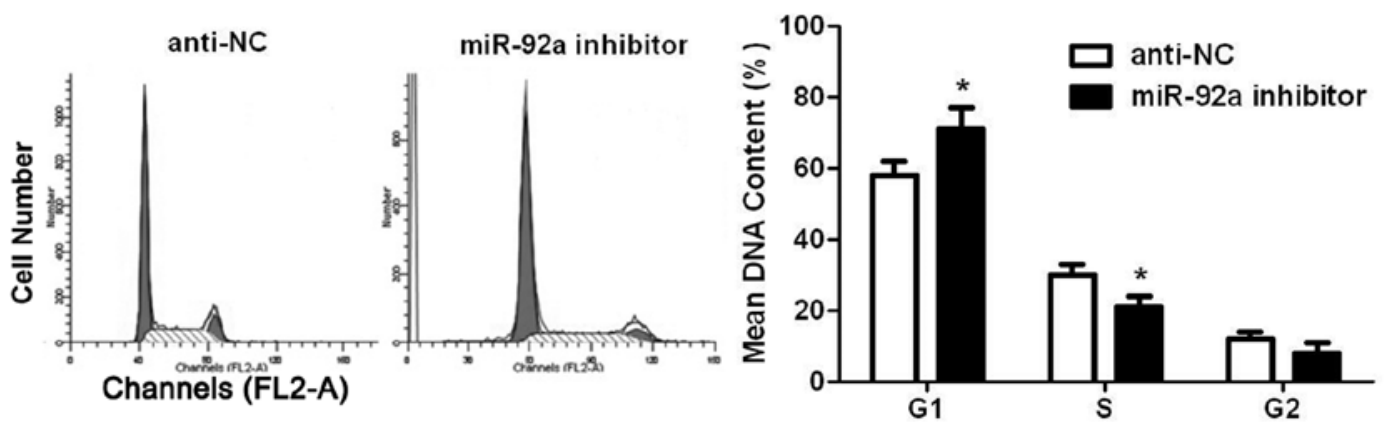

Figure 2. The effects of miR-92a inhibition on the proliferation, apoptosis and cell cycle progression of U2OS cells. (A) U2OS cells were transfected with miR-92a inhibitor and negative control inhibitor (anti-NC), respectively. miR-92a inhibitor significantly reduced the expression level of miR-92a in U2OS cells. ${ }^{*} \mathrm{P}<0.05$ by t-test. (B) miR-92a inhibition significantly decreased the proliferation of U2OS cells. ${ }^{*} \mathrm{P}<0.05$ by ANOVA. (C) miR-92a inhibition significantly increased the apoptosis of U2OS cells. " $\mathrm{P}<0.05$ by t-test. (D) miR-92a inhibition significantly inhibited the cell cycle progression of U2OS cells, as suggested by increased ratio of cells at G1 phase and decreased ratio of cells at $S$ phase. ${ }^{*} \mathrm{P}<0.05$ by t-test.

milk/TBST and were incubated with following antibodies at $4^{\circ}$ Covernight: PTEN antibody (Abcam, Cambridge, MA, USA),
p-AKT antibody (Ser473, Cell Signaling Technology, Danvers, MA, USA), AKT antibody (Cell Signaling Technology), mTOR 

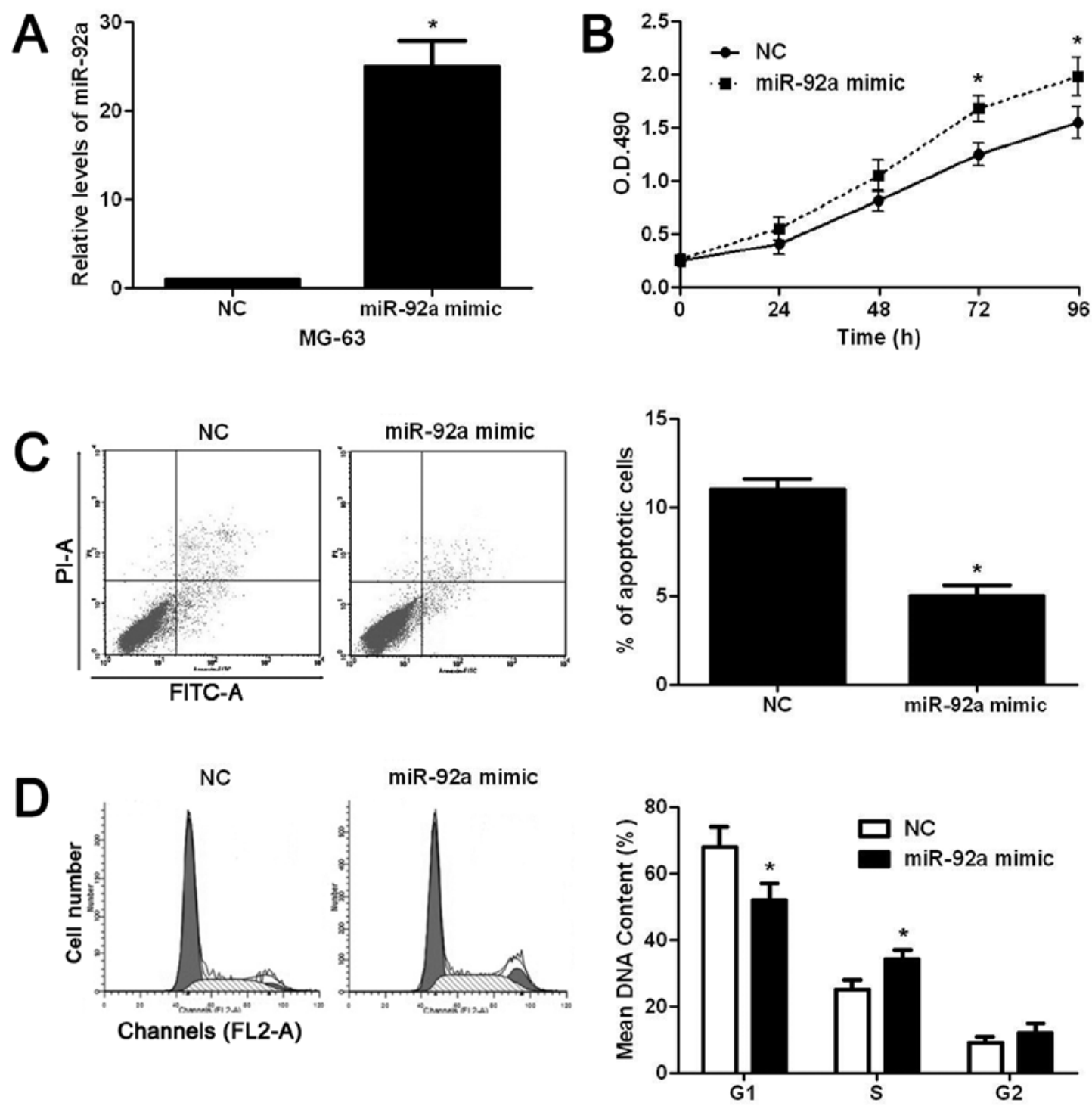

Figure 3. The effects of miR-92a overexpression on the proliferation, apoptosis and cell cycle progression of MG-63 cells. (A) MG-63 cells were transfected with miR-92a mimic and negative control mimic (NC), respectively. miR-92a mimic significantly increased the expression level of miR-92a in MG-63 cells. ${ }^{*} \mathrm{P}<0.05$ by t-test. (B) miR-92a overexpression significantly increased the proliferation of MG- 63 cells. ${ }^{\text {"P }}<0.05$ by ANOVA. (C) miR-92a overexpression significantly decreased apoptosis of MG-63 cells. " $\mathrm{P}<0.05$ by t-test. (D) miR-92a overexpression significantly promoted the cell cycle progression of MG-63 cells, as suggested by decreased ratio of cells at G1 phase and increased ratio of cells at $\mathrm{S}$ phase. ${ }^{*} \mathrm{P}<0.05$ by $\mathrm{t}$-test.

(Cell Signaling Technology), p-p27(Thr157, R\&D Systems, Inc., Minneapolis, MN, USA), p-MDM2(Ser166, Cell Signaling Technology), and GAPDH antibody (Proteintech, Chicago, IL, USA). Formed immunocomplexes between the targeted protein and primary antibodies were detected with secondary antibodies (GE Healthcare, Tokyo, Japan) and were visualized using ECL Prime system (GE Healthcare).

Luciferase reporter assay. Plasmids containing wild-type (wt) 3'-UTR of PTEN or the mutant (mt) PTEN 3'-UTR and corresponding miRNA vectors were transfected into U2OS cells using Lipofectamine 2000 based on manufacturer's protocol. At $48 \mathrm{~h}$ after transfection, U2OS cells were collected for measuring luciferase activity with the luciferase reporter assay system (Promega, Madison, WI, USA).

Statistical analysis. The experiments in this study were repeated at least three times. All data were collected and showed as mean \pm SEM. Statistical analysis including Pearson's Chi-squared test, Student's t-test, ANOVA, Kaplan-Meier analysis, Log-rank test and Spearman's correlation analysis were performed in this study with Graphpad (GraphPad Software, Inc., San Diego, CA, USA). The statistical significance level was set at $\mathrm{P}<0.05$.

\section{Results}

Increased level of miR-92a in OS is correlated with malignant clinicopathological features and poor prognosis of patients. We first evaluated the expression level of miR-92a in OS tissues. Compared with normal bone tissues, OS tissues showed significantly increased level of miR-92a $(\mathrm{P}<0.05$, Fig. 1A). This indicates that miR-92a probably plays an oncogenic role in OS. Then, we investigated the clinical significance and the prognostic value of miR-92a in OS. We divided all OS patients into two groups (miR-92a low group and miR-92a high group) 

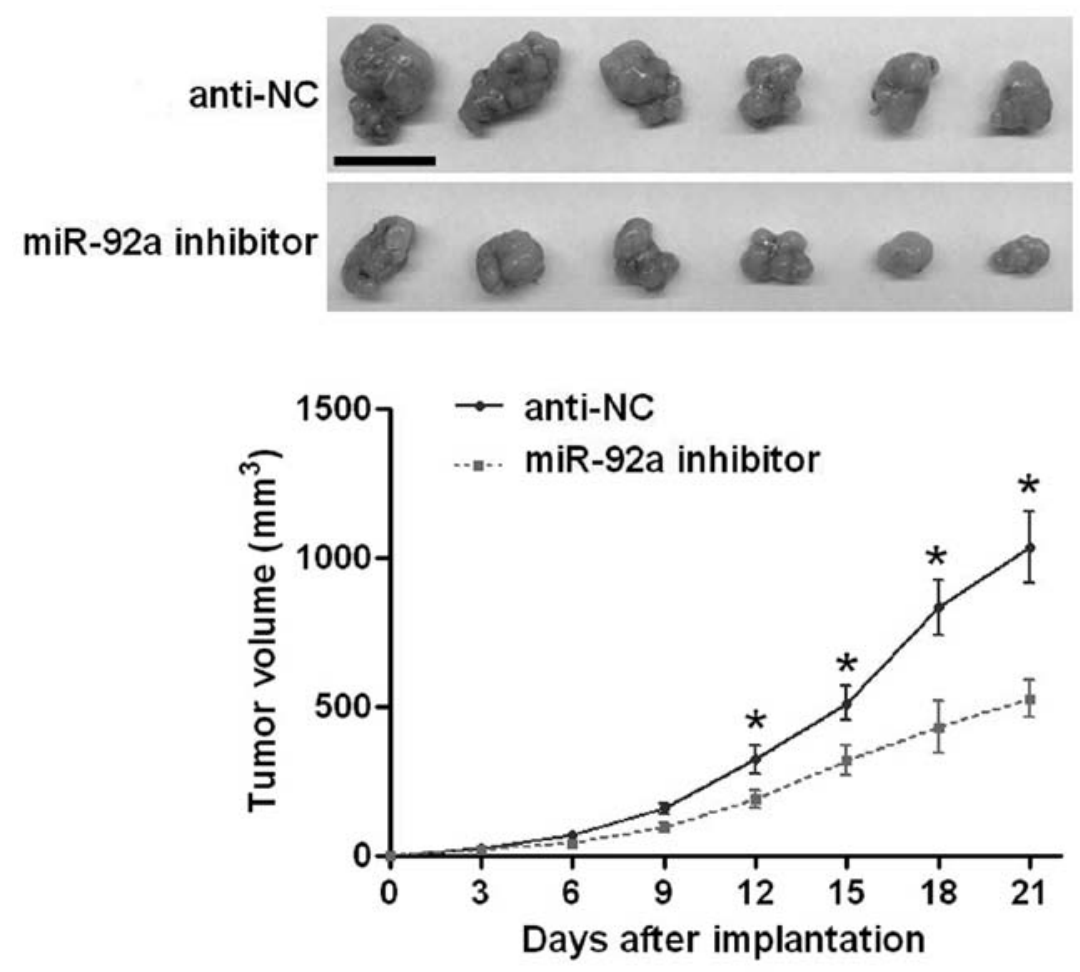

Figure 4. miR-92a inhibition significantly reduced the in vivo growth of U2OS cells. U2OS that were transduced with miR-92a inhibitor or negative control inhibitor were implanted into nude mice via subcutaneous injection. Tumor growth curves indicated that miR-92a knockdown significantly decreased the tumor growth of U2OS cells in nude mice. ${ }^{*} \mathrm{P}<0.05$ by ANOVA. Scale bar: $1 \mathrm{~cm}$.

using the median level of miR-92a expression as a cutoff value. Clinical association analysis (Table I) showed that compared with patients who had low expression level of miR-92a, patients with high level of miR-92a showed advanced TNM stage $(\mathrm{P}<0.001)$ and high $\mathrm{T}$ classification $(\mathrm{P}=0.003)$ and poor histological differentiation $(\mathrm{P}=0.026)$, indicating that miR-92a can act as promising biomarkers for OS patients.

miR-92a promotes proliferation and the cell cycle process, and reduces apoptosis of $O S$ cells. After confirming the elevated expression of miR-92a in OS, we examined the biological functions of miR-92a in OS cells. Increased proliferation, decreased apoptosis and deregulated cell cycle progression were critical for the malignant growth of cancer cells. Therefore, we examined whether miR-92a could promote the progression of OS by regulating these cellular functions. We first downregulated miR-92a expression level in U2OS cells with miR-92a inhibitor. As shown in Fig. 2A, miR-92a inhibitor significantly reduced the expression level of miR-92a in U2OS cells $(\mathrm{P}<0.05)$. Then, we used CCK8 assay to examine the alteration of proliferation after miR-92a knockdown in U2OS cells. miR-92a inhibition significantly reduced the proliferative ability of U2OS cells ( $\mathrm{P}<0.05$, Fig. $2 \mathrm{~B})$. The flow cytometry assay showed that miR-92a inhibition significantly increased the apoptotic rate of U2OS cells ( $\mathrm{P}<0.05$, Fig. 2C).

Furthermore, cell cycle assay showed that miR-92a inhibition prevented the cell cycle progression of U2OS cells as suggested by significantly increased ratio of cells at G1 phase and decreased ratio of cells at $\mathrm{S}$ phase $(\mathrm{P}<0.05$, Fig. 2D). On the contrary, miR-92a mimic significantly increased the level of miR-92a in MG-63 cells $(\mathrm{P}<0.05$, Fig. 3A). Subsequently,
miR-92a overexpression significantly increased the proliferation ( $\mathrm{P}<0.05$, Fig. 3B), decreased apoptosis $(\mathrm{P}<0.05$, Fig. 3C), and facilitated the cell cycle progression $(\mathrm{P}<0.05$, Fig. 3D) of MG-63 cells. After confirming the functional effects of miR-92a in vitro, we performed subcutaneous implantation model to test whether miR-92a could affect the growth of U2OS cells in vivo. As shown in Fig. 4, inhibiting miR-92a significantly reduced the growth of U2OS cells in nude mice $(\mathrm{P}<0.05$, Fig. 4). The above indicates that miR-92a can promote the growth of OS cells in vitro and in vivo.

PTEN is a downstream target of miR-92a in OS cells. To understand the underlying mechanisms for the functional influence of miR-92a on OS cell proliferation, apoptosis and cell cycle progression, we used public database (Targetscan) to search for potential downstream targets of miR-92a. As shown in Fig. 5A, the wt 3'-UTR of PTEN contained potential binding sites for miR-92a, indicating PTEN was a potential downstream target of miR-92a. Next, we examined the correlation between the level of PTEN mRNA and miR-92a expression in OS tissues. Our data indicated that the expression level of PTEN mRNA was negatively correlated with miR-92a expression in OS tissues ( $\mathrm{r}=-0.548, \mathrm{P}<0.05$, Fig. 5B). Then, we examined whether miR-92a overexpression could affect the expression level of PTEN mRNA and protein in OS cells. The results of qRT-PCR showed that miR-92a overexpression significantly reduced the expression level of PTEN mRNA in MG-63 cells $(\mathrm{P}<0.05$, Fig. 5C). Western blot results showed that miR-92a overexpression significantly reduced the PTEN protein level in MG-63 cells ( $\mathrm{P}<0.05$, Fig. 5D). Lastly, we performed luciferase reporter assay to determine whether miR-92a regulated the 
A
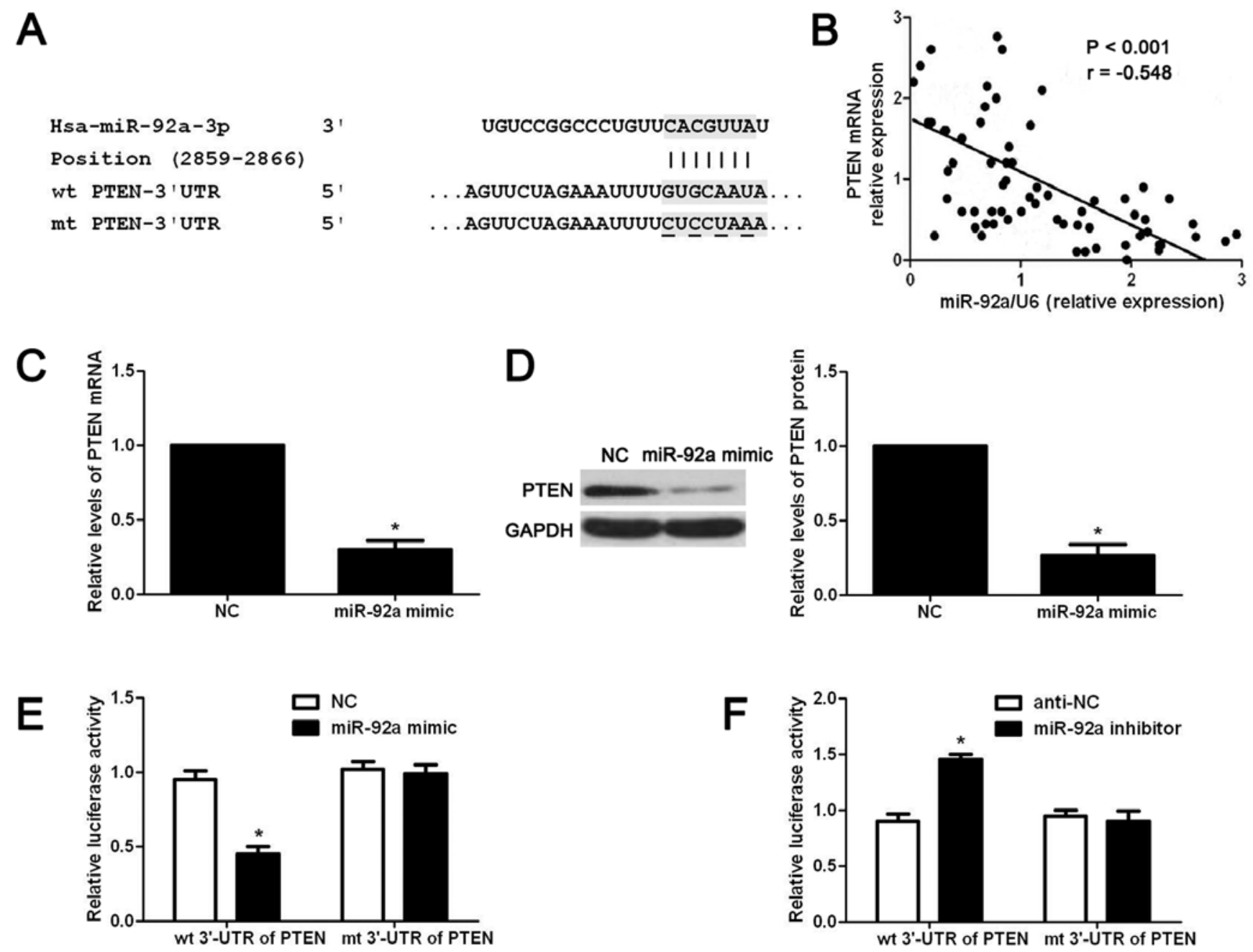

Figure 5. PTEN was a direct target of miR-92a. (A) The wild-type (wt) and mutant (mt) 3'-UTR of PTEN contained the complementary sequences of miR-92a. (B) The expression of PTEN mRNA was negatively correlated with the level of miR-92a in OS tissues. P $<0.05$ by Spearman's correlation analysis. (C) miR-92a overexpression significantly reduced the level of PTEN mRNA in MG-63 cells. ${ }^{*} \mathrm{P}<0.05$ by $\mathrm{t}$-test. (D) miR-92a overexpression significantly reduced the level of PTEN protein in MG-63 cells. "P<0.05 by t-test. (E) Overexpression of miR-92a decreased the luciferase activity of wt 3'-UTR of PTEN while had no effect on that of mt 3'-UTR of PTEN in U2OS cells. "P<0.05 by t-test. (F) Inhibition of miR-92a increased the luciferase activity of wt 3'-UTR of PTEN while had no effect on that of $\mathrm{mt} 3$ '-UTR of PTEN in U2OS cells. "P<0.05 by t-test.
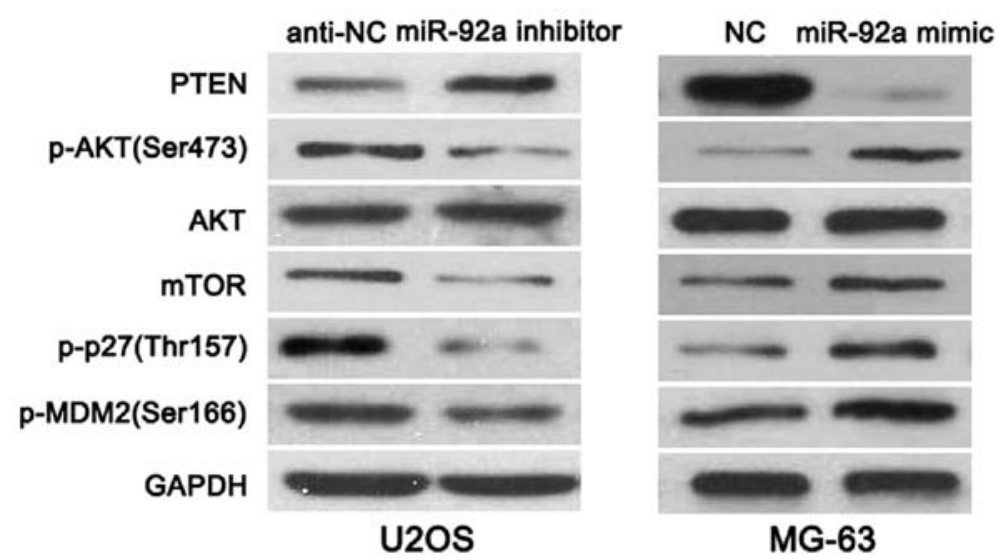

Figure 6. miR-92a regulated PTEN/AKT pathway in OS cells. miR-92a knockdown increased the level of PTEN and reduced the expressions of p-AKT(Ser473), mTOR, p-p27(Thr157) and p-MDM2(Ser166) in U2OS cells. While, overexpressing of miR-92a decreased PTEN level and led to increased expression of p-AKT(Ser473), mTOR, p-p27(Thr157) and p-MDM2(Ser166) in MG-63 cells.

expression of miR-92a through directly interacting with 3'-UTR of PTEN. As shown in Fig. 5E and F, overexpression of miR-92a significantly reduced the luciferase activity of wt 3'-UTR of PTEN (P<0.05, Fig. 5E) while miR-92a inhibition increased 
A
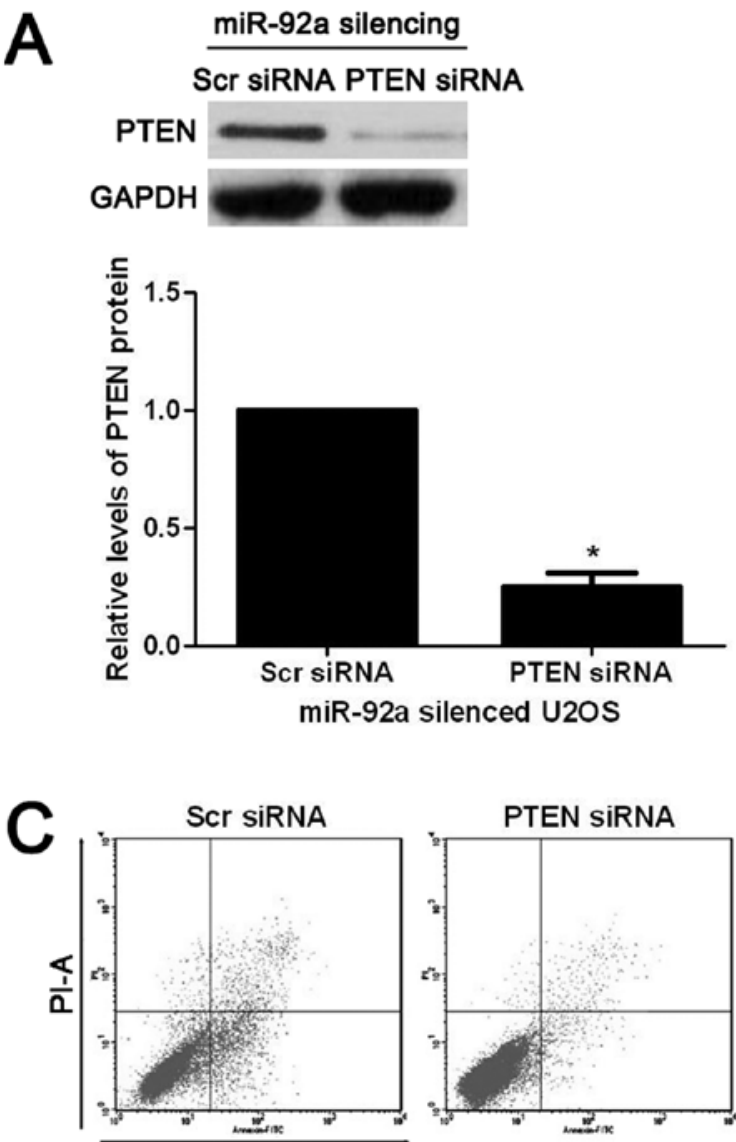

FITC-A

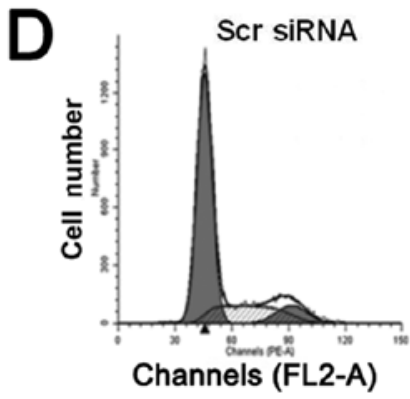

B
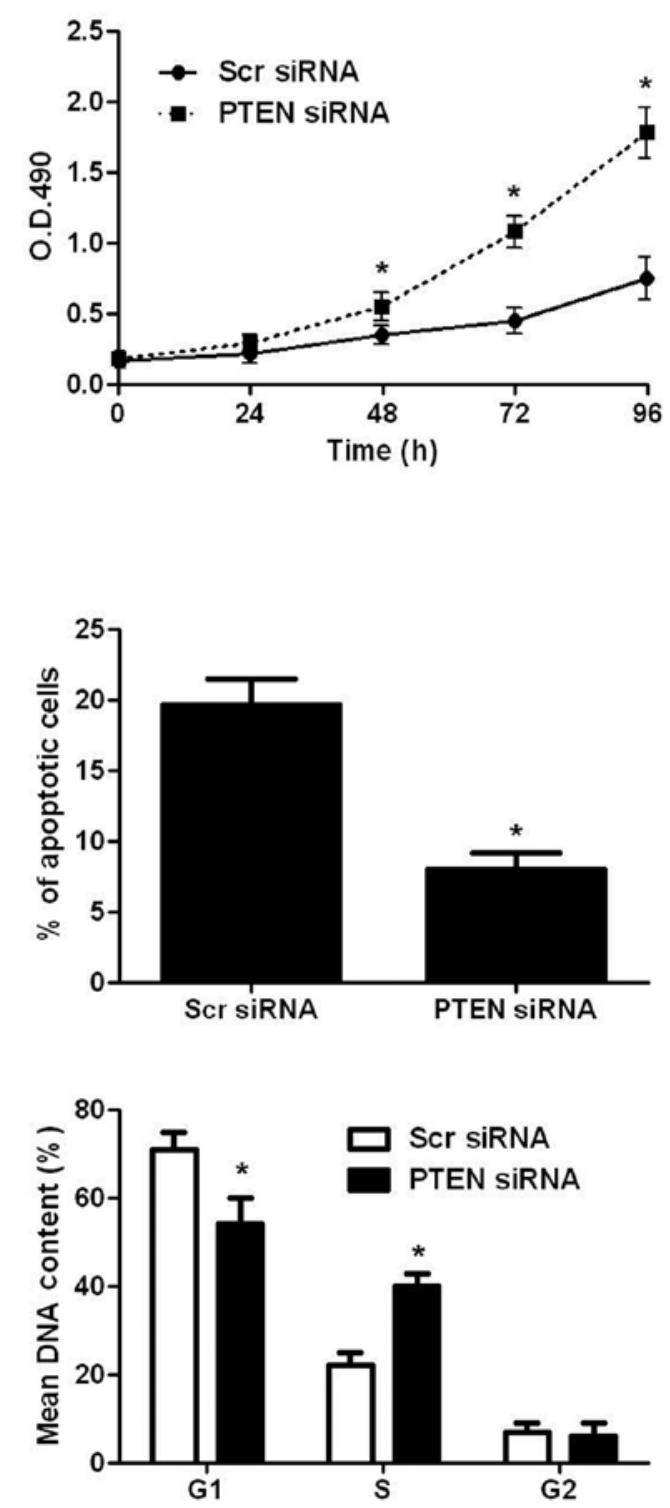

Figure 7. PTEN inhibition significantly reversed the functional effects of miR-92a knockdown on cell proliferation, apoptosis and cell cycle progression. (A) miR-92a silenced U2OS cells were transfected with PTEN siRNA and scrambled siRNA (scr siRNA), respectively. PTEN siRNA significantly reduced the expression of PTEN in miR-92a silenced U2OS cells. * $\mathrm{P}<0.05$ by t-test. (B) Knockdown of PTEN significantly increased the proliferation of miR-92a silenced U2OS cells. * $\mathrm{P}<0.05$ by ANOVA. (C) Knockdown of PTEN significantly decreased apoptosis of miR-92a silenced U2OS cells. ${ }^{*} \mathrm{P}<0.05$ by t-test. (D) Knockdown of PTEN significantly promoted the cell cycle progression of miR-92a silenced U2OS cells. ${ }^{*} \mathrm{P}<0.05$ by t-test.

that of wt 3'-UTR of PTEN (P<0.05, Fig. 5F). Furthermore, altering miR-92a level in OS cells did not affect the luciferase activity of mt 3'-UTR of PTEN (Fig. 5E and F).

miR-92a plays an oncogenic role by regulating PTEN/AKT pathway. PTEN, a versatile protein, inversely regulated PI3K/ AKT/mTOR signaling in cancer cells (18). Furthermore, PTEN/AKT pathway mediated the phosphorylation of p27(Thr157), AKT(Ser473) and MDM2(Ser166) (19). By regulating the expression and phosphorylation of these proteins, PTEN plays a tumor suppressive role in cancer cell proliferation, apoptosis and movements (19). To further explore the mechanisms involved in the function of miR-92a, OS cells that were transfected with corresponding miRNA vectors were subjected to western blot for PTEN, p-AKT(Ser473), AKT, mTOR, p-p27(Thr157) and p-MDM2(Ser166) expression. As shown in Fig. 6, miR-92a knockdown increased the level of PTEN and reduced the expression of p-AKT(Ser473), mTOR, p-p27(Thr157) and p-MDM2(Ser166) in U2OS cells. While, overexpressing of miR-92a decreased PTEN level and led to increased expression of p-AKT(Ser473), mTOR, p-p27(Thr157) and p-MDM2(Ser166) in MG-63 cells, thus indicating that miR-92a regulates PTEN/AKT pathway in OS cells.

PTEN is required for the biological functions of miR-92a in OS cells. To confirm whether PTEN was critical for the 
functional effects of miR-92a in OS cells, PTEN was knocked down by a specific siRNA in miR-92a silenced U2OS cells. PTEN siRNA significantly decreased the expression of PTEN in miR-92a silenced U2OS cells ( $\mathrm{P}<0.05$, Fig. 7A). Functional assays showed that knockdown of PTEN significantly reversed the effects of miR-92a silencing on U2OS cells with increased proliferation $(\mathrm{P}<0.05$, Fig. $7 \mathrm{~B})$, decreased apoptosis $(\mathrm{P}<0.05$, Fig. 7C), and enhanced cell cycle progression ( $\mathrm{P}<0.05$, Fig. 7D). Thus, PTEN is not only a downstream target but also mediator of miR-92a in OS.

\section{Discussion}

miR-92a was recently identified as a cancer-related microRNA. Studies of nasopharyngeal carcinoma (15), colorectal cancer (20), rectal cancer (21), gastric cancer (22), non-small cell lung cancer(23), hepatocellular carcinoma (14), cervival cancer (24) and ovarian cancer (25) showed that miR-92a played critical and different roles in the development and progression of these cancers. The expression of miR-92a is increased in OS cell lines compared to normal bone (17). However, the clinical values and function of miR-92a in OS has not been reported. Herein, we found that miR-92a was significantly increased in OS tissues. Increased expression of miR-92a was correlated with malignant clinical features and poor prognosis of OS patients, demonstrating that miR-92a potentially acts as a biomarker for OS.

miRNAs have been found to play various roles in cancer cells including cell proliferation, apoptosis, and metastasis and drug resistance (8). In this study, functional assays showed that miR-92a could promote the proliferation, inhibit apoptosis and contribute to cell cycle progression of OS cells. In this way, miR-92a promoted the in vivo growth of OS cells in nude mice. Therefore, we confirmed that miR-92a promotes the tumor growth of OS by regulating cell proliferation, apoptosis and cell cycle progression.

PTEN is a well-known tumor suppressor in human cancers and could regulate different biological functions of cancer cells including cell proliferation, apoptosis and metastasis $(26,27)$. In this study, we confirmed that miR-92a could directly target PTEN in OS cells with the following evidence: first, 3'-UTR of PTEN contained the complementary sequences for miR-92a; second, the expression of PTEN mRNA was negatively correlated with miR-92a level in OS tissues; third, miR-92a reduced the expression of PTEN mRNA and protein in OS cells; fourth, miR-92a inversely regulated the luciferase activity of wt 3'-UTR of PTEN rather than mt 3'-UTR of PTEN. PTEN/AKT pathway converts a subset of tumor suppressor proteins to oncogenic proteins or vice versa through multiple pathways (19). Herein, we disclosed that miR-92a regulated PTEN/AKT pathway and its downstream targets including mTOR, p-p27(Thr157) and p-MDM2(Ser166). Furthermore, functional assays confirmed that PTEN inhibition reversed the effects of miR-92a silencing on OS cells growth. Altogether, miR-92a promotes the tumor growth of OS probably by targeting the PTEN/AKT pathway.

A previous study reported that ZEB2 mRNA was a PTEN competitive endogenous RNA (ceRNA), which modulated PTEN protein levels in a microRNA-dependent manner including miR-92a (28). ZEB2 has been reported to be implicated in metastasis and epithelial-mesenchymal transition (EMT) in OS cells (29). Furthermore, miR-130a exerted promoting effects on metastatic behavior and EMT of OS cells through suppressing PTEN expression (30). Thus, it is worth to investigate crosstalk among ZEB2, PTEN and miR-92a in OS in further study.

Herein, we found that miR-92a expression was elevated in OS tissues and correlated with malignant clinicopathological features and reduced survival of OS patients. miR-92a played an oncogenic role in OS by promoting proliferation, inhibiting apoptosis and contributed to cell cycle progression. Mechanically, we showed that PTEN was a direct downstream target of miR-92a in OS and miR-92a exerted its functional influence on OS cells by regulating PTEN/AKT pathway.

\section{Acknowledgements}

This study was supported by the National Natural Science Foundation of China (grant nos. 81370976 and 81400904) and Medical-Engineering Joint Fund of Shanghai Jiaotong University (nos. YG2014MS41 and YG2016MS53).

\section{References}

1. Ottaviani G and Jaffe N: The epidemiology of osteosarcoma. In: Pediatric and Adolescent Osteosarcoma. Springer, New York, NY, pp3-13, 2009.

2. Laux CJ, Berzaczy G, Weber M, Lang S, Dominkus M, Windhager R, Nöbauer-Huhmann IM and Funovics PT: Tumour response of osteosarcoma to neoadjuvant chemotherapy evaluated by magnetic resonance imaging as prognostic factor for outcome. Int Orthop 39: 97-104, 2015.

3. Luetke A, Meyers PA, Lewis I and Juergens H: Osteosarcoma treatment - where do we stand? A state of the art review. Cancer Treat Rev 40: 523-532, 2014

4. Yates LA, Norbury CJ and Gilbert RJ: The long and short of microRNA. Cell 153: 516-519, 2013.

5. Gregory RI and Shiekhattar R: MicroRNA biogenesis and cancer. Cancer Res 65: 3509-3512, 2005.

6. Alvarez-Garcia I and Miska EA: MicroRNA functions in animal development and human disease. Development 132: 4653-4662, 2005.

7. Bartels CL and Tsongalis GJ: MicroRNAs: Novel biomarkers for human cancer. Clin Chem 55: 623-631, 2009.

8. Farazi TA, Hoell JI, Morozov P and Tuschl T: MicroRNAs in human cancer. In: MicroRNA Cancer Regulation. Springer, pp1-20, 2013.

9. Shen J, Stass SA and Jiang F: MicroRNAs as potential biomarkers in human solid tumors. Cancer Lett 329: 125-136, 2013.

10. Miao J, Wu S, Peng Z, Tania M and Zhang C: MicroRNAs in osteosarcoma: Diagnostic and therapeutic aspects. Tumour Biol 34: 2093-2098, 2013.

11. Volinia S, Calin GA, Liu CG, Ambs S, Cimmino A, Petrocca F, Visone R, Iorio M, Roldo C, Ferracin M, et al: A microRNA expression signature of human solid tumors defines cancer gene targets. Proc Natl Acad Sci USA 103: 2257-2261, 2006.

12. Ambs S, Prueitt RL, Yi M, Hudson RS, Howe TM, Petrocca F, Wallace TA, Liu CG, Volinia S, Calin GA, et al: Genomic profiling of microRNA and messenger RNA reveals deregulated microRNA expression in prostate cancer. Cancer Res 68: 6162-6170, 2008 .

13. Motoyama K, Inoue H, Takatsuno $\mathrm{Y}$, Tanaka F, Mimori K, Uetake H, Sugihara K and Mori M: Over- and under-expressed microRNAs in human colorectal cancer. Int J Oncol 34: 1069-1075, 2009.

14. Yang W, Dou C, Wang Y, Jia Y, Li C, Zheng X and Tu K: MicroRNA-92a contributes to tumor growth of human hepatocellular carcinoma by targeting FBXW7. Oncol Rep 34: 2576-2584, 2015.

15. Zhang H, Cao H, Xu D and Zhu K: MicroRNA-92a promotes metastasis of nasopharyngeal carcinoma by targeting the PTEN/AKT pathway. Onco Targets Ther 9: 3579-3588, 2016. 
16. Gougelet A, Pissaloux D, Besse A, Perez J, Duc A, Dutour A, Blay JY and Alberti L: Micro-RNA profiles in osteosarcoma as a predictive tool for ifosfamide response. Int J Cancer 129 : 680-690, 2011

17. Namløs HM, Meza-Zepeda LA, Barøy T, Østensen IH Kresse SH, Kuijjer ML, Serra M, Bürger H, Cleton-Jansen AM and Myklebost O: Modulation of the osteosarcoma expression phenotype by microRNAs. PLoS One 7: e48086, 2012.

18. Xiong J, Li Z, Zhang Y, Li D, Zhang G, Luo X, Jie Z, Liu Y, Cao Y, Le Z, et al: PRL-3 promotes the peritoneal metastasis of gastric cancer through the PI3K/Akt signaling pathway by regulating PTEN. Oncol Rep 36: 1819-1828, 2016.

19. Xie Y, Naizabekov S, Chen Z and Tokay T: Power of PTEN/AKT: Molecular switch between tumor suppressors and oncogenes. Oncol Lett 12: 375-378, 2016.

20. Zhou T, Zhang G, Liu Z, Xia S and Tian H: Overexpression of miR-92a correlates with tumor metastasis and poor prognosis in patients with colorectal cancer. Int J Colorectal Dis 28: 19-24, 2013.

21. Pelossof R, Chow OS, Fairchild L, Smith JJ, Setty M, Chen CT, Chen Z, Egawa F, Avila K, Leslie CS, et al: Integrated genomic profiling identifies microRNA-92a regulation of IQGAP2 in locally advanced rectal cancer. Genes Chromosomes Cancer 55: 311-321, 2016

22. Wu Q, Yang Z, Wang F, Hu S, Yang L, Shi Y and Fan D: MiR-19b/20a/92a regulates the self-renewal and proliferation of gastric cancer stem cells. J Cell Sci 126: 4220-4229, 2013.

23. Zhao J, Fu W, Liao H, Dai L, Jiang Z, Pan Y, Huang H, Mo Y, Li S, Yang G, et al: The regulatory and predictive functions of miR-17 and miR-92 families on cisplatin resistance of non-small cell lung cancer. BMC Cancer 15: 731, 2015.
24. Zhou C, Shen L, Mao L, Wang B, Li Y and Yu H: miR-92a is upregulated in cervical cancer and promotes cell proliferation and invasion by targeting FBXW7. Biochem Biophys Res Commun 458: 63-69, 2015.

25. Ohyagi-Hara C, Sawada K, Kamiura S, Tomita Y, Isobe A, Hashimoto K, Kinose Y, Mabuchi S, Hisamatsu T, Takahashi T, et al: miR-92a inhibits peritoneal dissemination of ovarian cancer cells by inhibiting integrin $\alpha 5$ expression. Am J Pathol 182: 1876-1889, 2013.

26. Ortega-Molina A and Serrano M: PTEN in cancer, metabolism, and aging. Trends Endocrinol Metab 24: 184-189, 2013.

27. Song MS, Salmena L and Pandolfi PP: The functions and regulation of the PTEN tumour suppressor. Nat Rev Mol Cell Biol 13: 283-296, 2012.

28. Karreth FA, Tay Y, Perna D, Ala U, Tan SM, Rust AG, DeNicola G, Webster KA, Weiss D, Perez-Mancera PA, et al: In vivo identification of tumor- suppressive PTEN ceRNAs in an oncogenic BRAF-induced mouse model of melanoma. Cell 147: 382-395, 2011.

29. Li M, Chen H, Zhao Y, Gao S and Cheng C: H19 Functions as a ceRNA in promoting metastasis through decreasing miR-200s activity in osteosarcoma. DNA Cell Biol 35: 235-240, 2016.

30. Chen J, Yan D, Wu W, Zhu J, Ye W and Shu Q: MicroRNA-130a promotes the metastasis and epithelial-mesenchymal transition of osteosarcoma by targeting PTEN. Oncol Rep 35: 3285-3292, 2016. 